\title{
Medication errors in a health care facility in southern Saudi Arabia
}

\author{
Sultan M Alshahrani ${ }^{1 *}$, Khaled M Alakhali ${ }^{1,2}$, Yaser Mohammed Al-Worafi $i^{3,4}$ \\ ${ }^{1}$ College of Pharmacy, King Khalid University, Abha, Saudi Arabia, ${ }^{2}$ College of Pharmacy, UCSI University, Malaysia, ${ }^{3}$ College \\ of Pharmacy, Ajman University, Ajman, United Arab Emirates, ${ }^{4}$ College of Pharmacy, University of Science and Technology, \\ Sana'a, Yemen
}

*For correspondence: Email: milhi1234@yahoo.com

Sent for review: 28 November 2018

Revised accepted: 18 April 2019

\begin{abstract}
Purpose: To identify medication errors at Aseer Central Hospital (ACH, Abha) in the southern province of Saudi Arabia.

Methods: A cross-sectional retrospective study was conducted by reviewing adult patients' records (> 15 years old) at ACH's inpatient and outpatients settings over an 8-week period in October and November 2015.

Results: We identified 113 medication errors, including 112 prescribing errors and 1 dispensing error. Most medication errors (91.2 \%) in this study were for inpatient prescriptions. The most common prescribing error was medication duplication (31.2\%) followed by missing patient identifying information (25\%).

Conclusion: Medication errors, mainly in inpatient prescriptions, have been fully identified at $\mathrm{ACH}$. Educational interventions such as workshops could help minimize and prevent medication errors.
\end{abstract}

Keywords: Medication errors, Prescribing, Dispensing, Guidelines

This is an Open Access article that uses a fund-ing model which does not charge readers or their institutions for access and distributed under the terms of the Creative Commons Attribution License (http://creativecommons.org/licenses/by/4.0) and the Budapest Open Access Initiative (http://www.budapestopenaccessinitiative.org/read), which permit unrestricted use, distribution, and reproduction in any medium, provided the original work is properly credited.

Tropical Journal of Pharmaceutical Research is indexed by Science Citation Index (SciSearch), Scopus, International Pharmaceutical Abstract, Chemical Abstracts, Embase, Index Copernicus, EBSCO, African Index Medicus, JournalSeek, Journal Citation Reports/Science Edition, Directory of Open Access Journals (DOAJ), African Journal Online, Bioline International, Open-J-Gate and Pharmacy Abstracts

\section{INTRODUCTION}

Medication errors (MEs) contribute to patient treatment outcomes $\mathrm{A}$ medication error refers to any avertable event that may result in occurrence of inappropriate medication use or patient harm while the medication is in the control of a healthcare professional, patient, or consumer" [1]. These errors can happen at any phase of the medication cycle, including prescription, dispensation, and patient use [1]. Medical prescriptions or orders that are transmitted from a prescriber to the pharmacy for dispensing should adhere to prescribing guidelines and should include the following information: authorized prescriber information, patient information, and medication-related information [2-4].

Dispensing errors are an important type of $\mathrm{ME}$ which are defined as any discrepancy between the written order or filled prescription including any written modifications made by the pharmacist during dispensing in compliance with the hospital policies [4]. Furthermore, the errors that occur during medication distribution or prescription selling to either the patient or his/her 
representative. [5]. In addition, MEs may refer to the error caught by a pharmacist during or after prescription validation. [6.7]. According to existing literature, an estimated $50 \%$ of MEs can be avoided [8], and they may cause severe injuries to patients The reported rate of serious patient injuries due to MEs ranges from 1 to $51.8 \%$. The estimated prevalence of death as a result of MEs is $30.5 \%$ deaths per year [9]. MEs are common in both developed and developing countries $[3,8,9]$. The objective of the current study was to investigate MEs at Aseer Central Hospital (ACH) in Abha, Saudi Arabia.

\section{METHODS}

\section{Study design and setting}

A cross-sectional retrospective study was conducted at $\mathrm{ACH}$, Abha during 8 weeks in October and November 2015. The patients' files were reviewed for adult patients (> 15 years old) who visited the hospital during that time period. Aseer Central Hospital (ACH) is the largest tertiary hospital (equipped with > 550 beds) in the southern region of Saudi Arabia that provides health care for population of Aseer, Jizan, and Najran cities and many rural villages.

\section{Definitions}

A prescribing error was defined as an error due to a prescribing decision or the prescription writing process, which unintentionally leads to significant (1) reduction in the probability of timely and effective treatment or (2) increase in the risk of patient harm compared to the generally accepted practice [10]. Dispensing errors in this study were those that occurred during prescription distribution or sale to a patient or patient agent [6].

\section{Data collection}

Prescribing- and dispensing-error evaluation checklists were developed based on literature review $[9,10]$. The dispensing-error evaluation checklist contained the following information: the date and time of the error, who made and identified the error, the type of the dispensing error, and the cause of the error.

\section{Sample size and sampling techniques}

A convenience sampling technique was used in this study. The data was collected from outpatient and inpatient setting during the study period. The files were reviewed once for all patients including the recurrent ones. The number of reviewed files for the study purpose during that period of time (n) was 386.

\section{Statistical analysis}

The data were descriptively analyzed and the data was reported as frequency (n) and the percentage (\%) for each type from. Then the data were represented using the Statistical Package for the Social Sciences (IBM SPSS) version 21 for Windows.

\section{Ethical approval}

An ethical clearance was granted from the college of pharmacy at King Khalid University in Aseer Region, Saudi Arabia (no. 37/9815, Date: 07/12/2014, 15/02/1436 Hijri). The data collection procedures were systematic and patients' information was remaining confidential along the study and during data analysis. This study was conducted in compliance with the World Medical Association [WMA] Declaration of Helsinki: Ethical principles for medical research involving human subjects amended by 59th WMA (no. PHRC/HC/11/13), 2013, Seoul, Korea. Furthermore, no personal information about patients, prescribers, or pharmacists was obtained.

\section{RESULTS}

We identified 113 MEs: 112 (91.9\%) were prescribing errors and $1(0.9 \%)$ was a dispensing error. Most MEs (91.2\%) in this study were for inpatient prescriptions, with the remainder (8.8\%) reported from the outpatient pharmacy. Table 1 summarizes the prescribing errors.

Table 1: Prescribing errors

\begin{tabular}{ll}
\hline Prescribing errors & $\mathbf{n}(\%)$ \\
\hline Missing patient identifier & $28(25)$ \\
Missing diagnosis & $7(6.25)$ \\
Inappropriate frequency and dose & $5(4.4)$ \\
Inappropriate duration & $5(4.4)$ \\
Therapeutic duplication & $12(10.7)$ \\
Missing prescriber information & $6(5.3)$ \\
Used prohibited abbreviation & $4(3.5)$ \\
Unclear prescription & $1(0.9)$ \\
Duplicate medications & $35(31.2)$ \\
Unnecessary medications & $9(8)$ \\
Total & 112 \\
\hline
\end{tabular}

\section{DISCUSSION}

The current study quantified MEs at $\mathrm{ACH}$ in Abha, Saudi Arabia. Duplicate medications were 
the most common prescribing error (31\%), followed by lack of patient information (25\%). A 2014 study conducted by Saghafi and Zargarzadeh regarding MEs in two hospitals in Isfahan, Iran, found that most common prescribing errors were drug name errors (34\%) and drug dose errors (27\%) [11]. In a 2015 Yemeni study, lack of patient information was the most common prescribing error [2]. Sheikh and colleagues reported that omission errors were most common $(77.7 \%)$ in a tertiary care hospital in India [12].

The prevalent type of error varies among studies and is affected by many factors, such as study design (i.e., self-reported by health care professionals or monitoring by data collectors) and site $[9,10,13,14]$. Notably, only one dispensing error $(0.9 \%)$ occurred during the study period. Al-Worafi and colleagues reported a $0.80 \%$ prevalence of dispensing errors in Ibb, Yemen but stated that they were self-reported, which could mean underreporting [15].

\section{Limitations of the study}

This retrospective study was conducted at a single health care facility in the southern province of Saudi Arabia. It would be better to perform more investigations on different hospitals in the region with different study designs to generalize the results and provide appropriate understanding about why MEs occur and will facilitate the appropriate design of ME checklists and interventions.

\section{CONCLUSION}

Many medication errors have been identified at $\mathrm{ACH}$. These are mostly related to inpatient prescriptions. Implementing educational interventions such as workshops and continuous medical education in the region is crucial in minimizing and preventing MEs in $\mathrm{ACH}$.

\section{DECLARATIONS}

\section{Acknowledgement}

We thank King Khalid University, Abha, Saudi Arabi for providing technical and administrative support and Aseer Central Hospital for allowing our researchers to perform the study.

\section{Conflict of interest}

No conflict of interest is associated with this work.

\section{Contribution of authors}

We declare that this work was done by the authors named in this article and all liabilities pertaining to claims relating to the content of this article will be borne by the authors. Sultan M. Alshahrani and Khaled Alakhali designed all the experiments and perform the study. Sultan M. Alshahrani, Khaled Alakhali, and Yaser Al-worafi wrote and revised the manuscript.

\section{Open Access}

This is an Open Access article that uses a funding model which does not charge readers or their institutions for access and distributed under the terms of the Creative Commons Attribution License (http://creativecommons.org/licenses/by/ 4.0) and the Budapest Open Access Initiative (http://www.budapestopenaccessinitiative.org/rea d), which permit unrestricted use, distribution, and reproduction in any medium, provided the original work is properly credited.

\section{REFERENCES}

1. Roy V, Gupta P, Srivastava S. Medication errors: causes \& prevention. Health Administrator 2006; 19(1): 60-64.

2. Al-Worafi YM, Patel RP, Zaidi ST, Alseragi WM, Almutairi MS, Alkhoshaiban AS, Ming LC. Completeness and Legibility of Handwritten Prescriptions at Sana'a, Yemen. Med Princ Pract 2018; 27(3): 290-292.

3. Davis L, Brunetti L, Lee EK, Yoon N, Cho SH, Suh DC. Effects of computerized physician order entry on medication turnaround time and orders requiring pharmacist intervention. Res Social Adm Pharm 2014; 10(5): 756-767.

4. Flynn EA, Barker KN, Carnahan BJ. National observational study of prescription dispensing accuracy and safety in 50 pharmacies. J Am Pharm Assoc 2003; 43: $191-200$.

5. Hoxsie DM, Keller AE, Armstrong EP. Analysis of community pharmacy workflow processes in preventing dispensing errors. J Pharm Pract 2006; 19: 124-130.

6. Flynn EA, Barker KN. Effect of an automated dispensing system on errors in two pharmacies. J Am Pharm Assoc 2006; 46(5): 613-615.

7. Nuckols TK, Bell DS, Liu H, Paddock SM, Hilborne LH. Rates and types of events reported to established incident reporting systems in two US hospitals. BMJ Qualit Safety 2007; 16(3): 164-168.

8. Anselmi ML, Peduzzi M, dos Santos CB. Errors in the administration of intravenous medication in Brazilian hospitals. J Clin Nurs 2007; 16(10): 1839-1847.

9. Al-Worafi YM. Dispensing errors observed by community pharmacy dispensers in lbb-Yemen. Asian J Pharm Clin Res 2018; 11(11): 478-481. 
10. Khaled M Alakhali, Shaik MA Ansari, Sirajudeen S. Alavudeen, Noohu A Khan. Medication errors at the outpatient pharmacy in a hospital in Aseer region, Kingdom of Saudi Arabia. Eur J Clin Pharm 2014; 16(2): 144-146.

11. Saghafi F, Zargarzadeh AH. Medication error detection in two major teaching hospitals: What are the types of errors? J Res Med Sci 2014; 19(7): 617-623.

12. Sheikh D, Mateti UV, Kabekkodu S, Sanal T. Assessment of medication errors and adherence to WHO prescription writing guidelines in a tertiary care hospital. Future J Pharmac Sci 2017; 3(1): 60-64.
13. Dean $B$, Barber $N$ and Schachter $M$. What is a prescribing error. BMJ Quality \& Safety 2000; 9(4): 232237.

14. Donchin Y, Gopher D, Olin M, Badihi Y, Biesky MR, Sprung CL, Pizov R, Cotev S. A look into the nature and causes of human errors in the intensive care unit. Qual Saf Health Care 2003; 12(2): 143-148.

15. Al-Worafi YM. Dispensing errors observed by community pharmacy dispensers in lbb-Yemen. Asian J Pharm Clin Res 2018; 11(11): 478-481. 